\title{
Endovascular Stent Grafting in Posttraumatic Tibial Artery Pseudoaneurysms: Case Report
}

\author{
1 Department of Imaging Sciences and Interventional Radiology, Sree \\ Chitra Tirunal Institute of Medical Sciences and Technology, \\ Trivandrum, Kerala, India \\ 2 Neurointervention Center, Department of Imaging Sciences and \\ Interventional Radiology, Sree Chitra Tirunal Institute of Medical \\ Sciences and Technology, Trivandrum, Kerala, India \\ J Clin Interv Radiol ISVIR 2017;1:61-64.
}

Sadananda Arjun Pisharody ${ }^{1}$ Santhosh Kumar Kannath ${ }^{2}$ Jayadevan Enakshy Rajan ${ }^{2}$

\begin{abstract}
Address for correspondence Santhosh Kumar Kannath, MD, PDCC, Neurointervention Center, Department of Imaging Sciences and Interventional Radiology, Sree Chitra Tirunal Institute of Medical Sciences and Technology, Trivandrum 695011, Kerala, India (e-mail: drsanthoshkannath@gmail.com).
\end{abstract}

\begin{abstract}
Keywords

- pseudoaneurysm

- stent graft

- endovascular

Infrapopliteal pseudoaneurysms are rare and usually occur secondary to trauma. Though there are no consensus regarding the management of the same, endovascular treatment has become an alternate option to open surgical management. In this report, we describe two cases of posttraumatic infrapopliteal pseudoaneurysms involving crural arteries. Both the cases were managed successfully with endovascular stent graft placement with complete exclusion of pseudoaneurysm. Our results show that endovascular stent grafting is a good alternative to open repair in infrapopliteal pseudoaneurysms, although long-term efficacy of this approach needs to be established.
\end{abstract}

\section{Introduction}

Aneurysms of infrapopliteal arteries are uncommon. Most often they are pseudoaneurysms secondary to orthopaedic or iatrogenic trauma. Open surgical repair has been the standard mode of management; however, recently there is a growing trend in favor of endovascular repair. ${ }^{1}$ In this article, we describe two cases of posttraumatic infrapopliteal pseudoaneurysms that were managed successfully with endovascular covered stent placement.

\section{Case Description}

\section{Case 1}

A 54-year-old patient who had sustained fracture of both tibia and fibula following a road traffic accident 18 months ago presented with progressively enlarging painful swelling in the right lower leg on the lateral aspect. There was ulceration of the overlying skin with a history of episodic acute bleeding 1 week prior to admission. On examination, tense pulsatile swelling was noted on the right leg, predominantly in the anterolateral aspect. The patient was evaluated with a Doppler ultrasonogram followed by computed tomography (CT) angiography from an outside institution, which revealed pseudoarthrosis of shaft of tibia and fibula with evidence of a pseudoaneurysm in relation to right anterior tibial artery (ATA).

Patient was initiated on clopidogrel $75 \mathrm{mg}$ and Ecospirin (USV Limited) $150 \mathrm{mg}, 5$ days prior to the intervention. Right lower limb angiogram was performed under local anesthesia with an antegrade approach through right common femoral artery. Diagnostic angiograms revealed a large pseudoaneurysm at midcalf region communicating with right ATA (-Fig. 1A). Luminal segment of pseudoaneurysm measured approximately $4.6 \times 3.2 \mathrm{~cm}$ and the neck measured approximately $2.3 \mathrm{~mm}$. The diameter of the parent artery was $3 \mathrm{~mm}$. The other tibial arteries appeared patent. Following this, a $6 \mathrm{~F}$ guiding catheter was placed in the distal popliteal artery and a $3 \times 20 \mathrm{~mm}$ Prograft (Vascular Concepts, Essex, United Kingdom) balloon expandable covered stent over a 0.014inch wire was positioned across the neck of the pseudoaneurysm. After confirming position, this stent was expanded to its nominal diameter, and a postdeployment angiogram showed good forward flow across it with complete exclusion of pseudoaneurysm ( - Fig. 1B, C). The patient developed a small puncture site and scrotal hematoma, which were managed conservatively. Significant resolution of hematoma was 

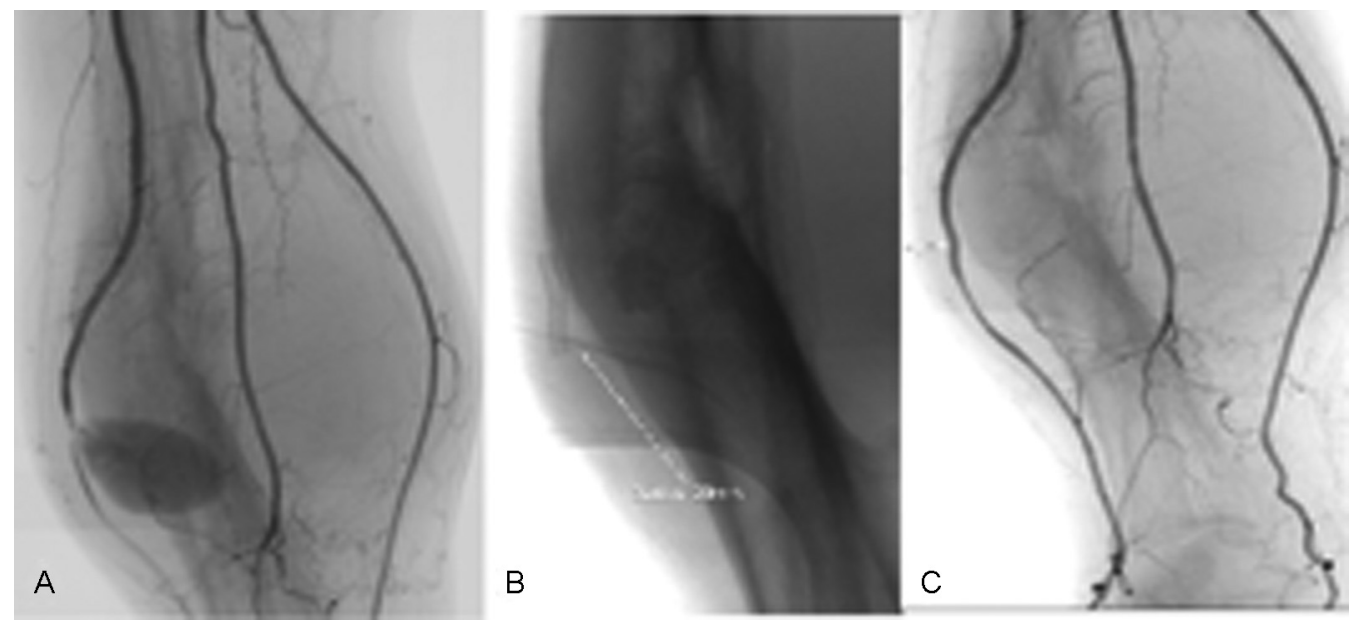

Fig. 1 (A) A large pseudoaneurysm is seen arising from the midsegment of the right anterior tibial artery. (B) A covered stent graft is placed across the neck of aneurysm and (C) a final check angiogram shows complete exclusion of the aneurysm with patent anterior tibial artery.

observed at 3 months follow-up, and Doppler ultrasound showed patent stent graft with good distal run-off.

\section{Case 2}

A 26-year-old male presented with complaints of rapidly increasing pain and swelling of the left upper calf region for the previous 1 month, following the removal of orthopaedic implant placed for tibial shaft fracture. On examination, there was an ill-defined swelling in the proximal calf region, which showed increased warmth and thrill on palpation. Distal pulses were feeble. CT angiogram ( - Fig. 2A) revealed the presence of a large peripherally thrombosed pseudoaneurysm $(10 \times 7.5 \times 4 \mathrm{~cm})$ in relation to the tibioperoneal trunk with evidence of dilated early draining veins within the vicinity of aneurysm, suggestive of an associated arteriovenous fistula (AVF).
Dual antiplatelet medications were initiated 5 days prior to the procedure. Angiography was performed through left antegrade femoral route, which confirmed the presence of pseudoaneurysm and AVF from the proximal tibioperoneal trunk (-Fig. 2B). The rent of aneurysm measured $9 \mathrm{~mm}$ and the fistulous communication arose from the rupture of aneurysm into the adjacent tibial veins. The parent artery diameter was $3.3 \mathrm{~mm}$. A $6 \mathrm{~F}$ guiding catheter was placed in the popliteal artery, and a $4 \times 30 \mathrm{~mm}$ Prograft covered stent was navigated over a 0.014-inch micro guidewire and deployed in the parent artery across the dehiscence, sealing the communication with the pseudoaneurysm. Postdeployment check angiogram revealed complete exclusion of the pseudoaneurysm with no evidence of any early draining veins (-Fig. 2C). In the postoperative period, there was significant pain relief along with restoration of distal pulses. After 2 months, there was

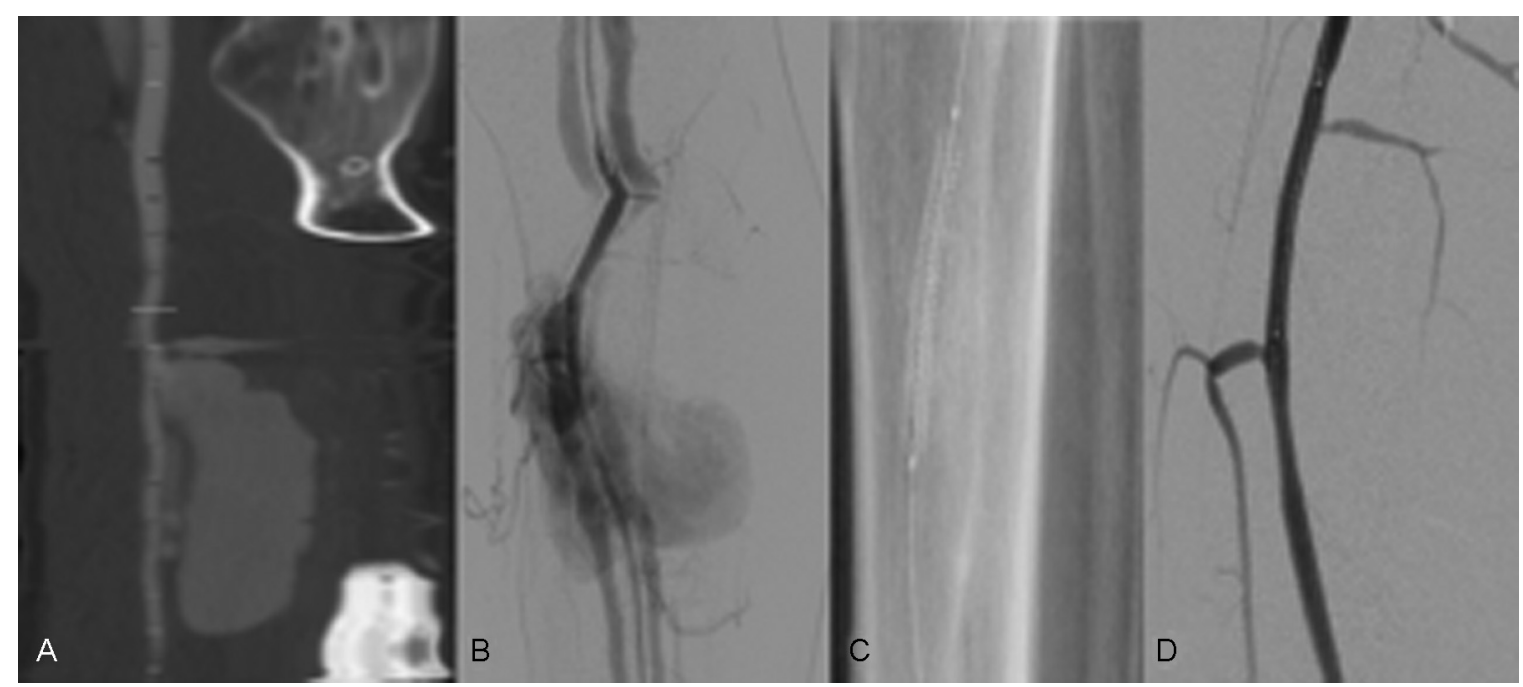

Fig. 2 (A) Computed tomography angiogram shows a large irregular aneurysm arising from the left tibioperoneal trunk. (B) Immediate appearance of tibial veins is noted, suggesting a coexistent arteriovenous fistula. (C) The rent is sealed using a covered stent graft and (D) final angiogram reveals patent tibial artery with obliteration of aneurysm. 
significant reduction in swelling, and Doppler ultrasound showed patent stent with no evidence of pseudoaneurysm or fistula.

\section{Discussion}

Pseudoaneurysm or false aneurysm is defined as aneurysm that is not contained by all three layers of a normal artery. These are formed as a result of persistent communication between an artery and the adjacent blood-filled cavity. Due to continuous arterial pressure, it can increase in size over time and often presents with a pulsatile swelling. Associated pain may arise due to compression of adjacent structures. Vascular injury involving infrapopliteal region following bone fractures and orthopaedic procedures are relatively rare; however, there are multiple case reports of pseudoaneurysm following orthopaedic trauma. ${ }^{2}$ Often, the diagnosis of pseudoaneurysm is delayed, as in our first case, mistaking it for a hematoma, which is very much common in orthopaedic practice. ${ }^{3}$

The diagnosis is often made by an initial Doppler ultrasonography, which has got a high sensitivity and specificity in experienced hands. CT angiography is usually done to confirm the diagnosis, which has got a sensitivity of $90 \%$ and specificity of $100 \%$ in extremity trauma. ${ }^{4}$ With the recent advances in magnetic resonance (MR) imaging, MR angiography provides excellent three-dimensional images similar to CT without administration of contrast. However, MR is not generally preferred because of long imaging time and artifacts. Angiography remains the gold standard investigation to diagnose pseudoaneurysm and AVF with a diagnostic accuracy of nearly $98 \%$. However, the angiography is rarely performed as a diagnostic procedure alone and is usually done as a part of definitive endovascular repair.

Management options include both surgical and endovascular approaches. Surgery has been the traditional mode of treatment for tibial artery aneurysms; however, it is often challenging, as the tissue planes are often distorted due to previous surgeries or large hematomas. ${ }^{5}$ Recently, there is growing evidence to support the use of endovascular techniques; however, the literature on its application in infrapopliteal vessels is limited. ${ }^{6-8}$ Endovascular treatment options include ultrasound-guided percutaneous thrombin injection, placement of covered stent or coil embolization, and trapping of involved artery. ${ }^{9}$ The choice of endovascular technique depends upon the vessel involved and the width of the neck, as well as the expendable nature of the artery. Though percutaneous thrombin injection is a less invasive option, there are concerns of distal thromboembolism or deep venous thrombosis in wide neck aneurysms and coexistent AVFs, respectively. ${ }^{10}$ The affected artery could be sacrificed if other tibial arteries are normal, and a collateral supply to the involved arterial territory is demonstrated. Covered stent placement is technically easy and does not involve the risk of parent artery occlusion or distal embolization. Since the diameter of the tibial arteries is small, the coronary covered graft would be generally sufficient for the reconstruction of the artery. Balloon expandable stent allows accurate placement of stent across the rent, and a good apposition could be achieved due to their high radial strength. Adequate platelet suppression is mandatory to prevent acute in-stent thrombosis and to preserve long-term patency, and the guidelines for its administration are often adopted from cardiology literature. ${ }^{11}$ The dual antiplatelet drugs are initiated 5 days prior to the procedure, which are continued for at least 3 months, and thereafter aspirin is continued lifelong. However, in emergent situations, loading dose of clopidogrel or intravenous antithrombotic drugs such as tirofiban should be considered.

Long-term results of covered stent placement in pseudoaneurysm, especially infrapopliteal arteries, are not well studied. Gratl et al ${ }^{12}$ reported that eight of the nine patients who underwent stent graft placement for pseudoaneurysm had the grafts occluded during a mean follow-up period of 7 months. All the patients had significant peripheral vascular disease of the parent arteries and most of them were not adequately anticoagulated. In one of our cases, the stent remained patent at 2 years of follow-up, suggesting a durable treatment outcome. It can be assumed that the results will be better compared with peripheral occlusive vascular disease because most of the patients are younger and without significant other comorbidities.

\section{Conclusion}

Infrapopliteal pseudoaneurysms are rare but pose diagnostic as well as therapeutic challenge. Covered stent placement using endovascular technique is an easy and feasible treatment option with excellent success rate.

\section{Contributions of Authors}

Sadananda Arjun Pisharody contributed in data analysis, data interpretation, manuscript preparation, and critical revision, and Santhosh Kumar Kannath and Jayadevan Enakshy Rajan contributed in concept and design, data analysis, data interpretation, manuscript preparation, and critical revision.

\section{Conflicts of Interest}

The authors have no personal or financial conflict of interest to disclose.

\section{References}

1 Joglar F, Kabutey NK, Maree A, Farber A. The role of stent grafts in the management of traumatic tibial artery pseudoaneurysms: case report and review of the literature. Vasc Endovascular Surg 2010;44(5):407-409

2 van Hensbroek PB, Ponsen KJ, Reekers JA, Goslings JC. Endovascular treatment of anterior tibial artery pseudoaneurysm following locking compression plating of the tibia. J Orthop Trauma 2007;21(4):279-282

3 Ramavath AL, Cornish JA, Ganapathi M, Williams DT. Missed diagnosis of ankle pseudoaneurysm following ankle arthroscopy: a case report. Cases J 2009;2:162 
4 Foster BR, Anderson SW, Soto JA. CT angiography of extremity trauma. Tech Vasc Interv Radiol 2006;9(4):156-166

5 Darbari A, Tandon S, Chandra G, et al. Post-traumatic peripheral arterial pseudoaneurysms: our experience. Ind J Thorac Cardiovasc Surg 2006;22:182-187

6 Parodi JC, Schönholz C, Ferreira LM, Bergan J. Endovascular stentgraft treatment of traumatic arterial lesions. Ann Vasc Surg 1999; 13(2):121-129

7 De Roo RA, Steenvoorde P, Schuttevaer HM, Den Outer AJ, Oskam J, Joosten PP. Exclusion of a crural pseudoaneurysm with a PTFEcovered stent-graft. J Endovasc Ther 2004;11(3):344-347

8 Halabi AR, Kandzari DE. The thrill is gone: catheter-based exclusion of a posttraumatic arteriovenous fistula with a covered stent graft. Catheter Cardiovasc Interv 2005;66(1):27-33
9 Spirito R, Trabattoni P, Pompilio G, Zoli S, Agrifoglio M, Biglioli P. Endovascular treatment of a post-traumatic tibial pseudoaneurysm and arteriovenous fistula: case report and review of the literature. J Vasc Surg 2007;45(5):1076-1079

10 Webber GW, Jang J, Gustavson S, Olin JW. Contemporary management of postcatheterization pseudoaneurysms. Circulation 2007; 115(20):2666-2674

11 Levine GN, Bates ER, Bittl JA, et al. 2016 ACC/AHA Guideline Focused Update on Duration of Dual Antiplatelet Therapy in Patients With Coronary Artery Disease: A Report of the American College of Cardiology/American Heart Association Task Force on Clinical Practice Guidelines. J Am Coll Cardiol 2016;68(10):1082-1115

12 Gratl A, Klocker J, Glodny B, Wick M, Fraedrich G. Treatment options of crural pseudoaneurysms. Vasa 2014;43(3):209-215 